\title{
Técnica CAD/CAM comparado con técnica convencional en pacientes con indicación de restauración indirecta unitaria posterior.
}

\section{CAD/CAM versus conventional techniques for unitary posterior indirect restoration.}

\begin{abstract}
Orlando Fierro ${ }^{1,2}$, Andrés Verdugo ${ }^{1,2}$, Bárbara Barrientos ${ }^{1,2,3^{*}}$
1. Escuela de Odontología, Facultad de Medicina, Pontificia Universidad Católica de Chile, Santiago, Chile

2. Proyecto Epistemonikos, Santiago, Chile

3. Departamento de Rehabilitación oral, Facultad de Medicina, Pontificia Universidad Católica de Chile, Santiago, Chile

* Correspondencia Autor: Bárbara Barrientos | Dirección: Centro Evidencia UC, Pontificia Universidad Católica de Chile, Diagonal Paraguay 476, Santiago, Chile | E-mail: bbarrientos@uc.cl

RESUMEN

Introducción: La confección de restauraciones indirectas ha sido tradicionalmente realizada mediante técnicas convencionales de laboratorio, la cual involucra la realización de una serie de pasos y la necesidad de que el paciente asista a varias sesiones clínicas. En las últimas décadas ha sido incorporada la técnica CAD/CAM, la cual ha simplificado la confección de restauraciones indirectas. Es necesario comparar el desempeño clínico de ambas técnicas. Métodos: Realizamos una búsqueda en Epistemonikos, la mayor base de datos de revisiones sistemáticas en salud, la cual es mantenida mediante el cribado de múltiples fuentes de información, incluyendo MEDLINE, EMBASE, Cochrane, entre otras. Extrajimos los datos desde las revisiones identificadas, analizamos los datos de los estudios primarios, realizamos un metaanálisis y preparamos una tabla de resumen de los resultados utilizando el método GRADE. Resultados y conclusiones: Identificamos tres revisiones sistemáticas que en conjunto incluyeron ocho estudios primarios, de los cuales, cinco corresponden a ensayos aleatorizados. Concluimos que la técnica CAD/ CAM probablemente aumenta la falla de la restauración y la pérdida de retención. Por otra parte, podría disminuir levemente la pérdida de vitalidad y tener nula o poca diferencia en el riesgo de extracción dentaria, riesgo de caries secundaria y cambio de color superficial, pero la certeza de la evidencia es baja.
\end{abstract}

\section{ABSTRACT}

Introduction: Conventional laboratory techniques for indirect restoration involve multiple stages and several clinical sessions for patients. In the last decades, the CAD/ CAM technique has been incorporated in clinical practice, simplifying the fabrication process of indirect restorations. A comparative assessment of the clinical performance of both techniques is required. Methods: We searched in Epistemonikos, the largest database of systematic reviews in health, which is maintained by screening multiple information sources, including MEDLINE, EMBASE, Cochrane, among others. We extracted data from the systematic reviews, reanalyzed data of primary studies, conducted a meta-analysis and generated a summary of findings table using the GRADE approach. Results and conclusions: We identified three systematic reviews including eight studies overall, of which five were randomized trials. We conclude that CAD/CAM technique probably increase the risk of restoration failure and loss of retention. On the other hand, CAD/CAM technique may slightly reduce the risk of loss of vitality and may make little or no difference to the risk of tooth extraction, risk of secondary caries and superficial color change, but the certainty of the evidence is low.

\section{KEY WORDS}

Conventional technique; Heat-pressing technique; Casting technique; Slip casting technique; CAD/CAM, CEREC, Epistemonikos, GRADE.

Int. J. Inter. Dent Vol. 13(3); 207-211, 2020. 


\section{PROBLEMA}

Las restauraciones indirectas se utilizan para el tratamiento de caries, fracturas dentarias u otros desórdenes asociados a pérdidas de tejido dentario donde no está indicado el uso de restauraciones directas ${ }^{[1]}$. Estas tradicionalmente han sido confeccionadas mediante técnicas que incorporan una serie de pasos clínicos y de laboratorio, por lo que se requiere de una mayor cantidad de sesiones. Frente a esto, se introduce el sistema CAD/CAM al mercado y se abre paso a la odontología digital.

Computer Aided Design and Computer Aided Manufacturing (CAD/CAM) corresponde a un sistema digital usado en odontología restauradora en el que un software permite realizar un scanner intraoral y posterior diseño de la restauración (CAD), que guiará los movimientos tridimensionales de las fresas maquinadas que tallaran la restauración (CAM). Algunas ventajas de estos sistemas son el ahorro del tiempo en la confección de restauraciones, permitiendo incluso ser cementadas en la misma sesión y la disminución de costos instrumentales a largo plazo ${ }^{[2]}$. Por otro lado, el scanner intraoral (Computer Aided Design) disminuye la incomodidad del paciente respecto de la toma de impresión con elastómeros, además de eliminar el riesgo de que este registro sufra algún tipo de distorsión ${ }^{[3]}$. A pesar de resolver estas problemáticas, los sistemas CAD/CAM poseen desventajas. La necesidad de una gran inversión inicial y curva de aprendizaje para su utilización limitan su uso ${ }^{[4]}$. Además, los tallados previos al scanner intraoral deben cumplir el requisito de no posicionar sus terminaciones marginales subgingivales ${ }^{[5]}$.

Sin embargo, no existe claridad sobre el desempeño clínico de las técnicas CAD/CAM en comparación a la técnica convencional en restauraciones indirectas posteriores unitarias.

\section{MÉTODOS}

Realizamos una búsqueda en Epistemonikos, la mayor base de datos de revisiones sistemáticas en salud, la cual es mantenida mediante búsquedas en múltiples fuentes de información, incluyendo MEDLINE, EMBASE, Cochrane, entre otras. Extrajimos los datos desde las revisiones identificadas y analizamos los datos de los estudios primarios. Con esta información, generamos un resumen estructurado denominado FRISBEE (Friendly Summaries of Body of Evidence using Epistemonikos), siguiendo un formato preestablecido, que incluye mensajes clave, un resumen del conjunto de evidencia (presentado como matriz de evidencia en Epistemonikos), metanálisis del total de los estudios cuando sea posible, una tabla de resumen de resultados con el método GRADE y una sección de otras consideraciones para la toma de decisión

\section{Mensajes clave}

- La técnica CAD/CAM probablemente aumenta la falla y la pérdida de retención de la restauración

- La técnica CAD/CAM podría resultar en poca o nula diferencia el riesgo de extracción dentaria, riesgo de caries secundario y cambio de color superficial (certeza de la evidencia baja).

-Técnica CAD/CAM podría disminuir levemente la pérdida de vitalidad (certeza de la evidencia baja)

\section{Acerca del conjunto de evidencia para esta pregunta}

\begin{tabular}{|c|c|}
\hline Cuál es la evidencia & $\begin{array}{l}\text { Encontramos tres revisiones } \\
\text { sistemáticas } \\
\text { estudi, [8], [9] que incluyeron primarios }{ }^{[10], ~[11], ~[12], ~[13], ~[14], ~[15], ~[16], ~} \\
{[17], \text { de los cuales, cinco son ensayos }} \\
\text { aleatorizados } \\
{[10],[11], \text { [12], [13], [14]. }}\end{array}$ \\
\hline $\begin{array}{l}\text { Véase matriz de evidencia } \\
\text { en Epistemonikos más } \\
\text { abajo. }\end{array}$ & $\begin{array}{l}\text { Esta tabla y el resumen en general } \\
\text { se basan en estos últimos, dado } \\
\text { que los estudios observacionales no } \\
\text { aumentaban la certeza de la evidencia } \\
\text { existente, ni entregaban información } \\
\text { adicional relevante. }\end{array}$ \\
\hline
\end{tabular}

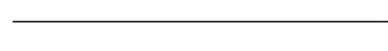

Cuatro ensayos ${ }^{\text {10], [11], [12], [13] incluyeron }}$ pacientes adultos que requerían al menos una restauración indirecta unitaria sobre un diente permanente posterior. Uno de los ensayos ${ }^{[14]}$ no reportó el tipo de pacientes incluidos en el ensayo.
Qué tipo de pacientes incluyeron los estudios*

Qué tipo de intervenciones incluyeron los estudios*

Qué tipo de desenlaces midieron
De los cinco ensayos, tres reportaron la edad promedio de los pacientes, variando entre $37,42,45$ años $^{[11], \text {, [12], [13] }}$ Dos ensayos ${ }^{[10], ~[14]}$ no reportaron esta información.,

De los cinco ensayos incluidos, cuatro[10] [11], [12], [13] incluyeron pacientes hombres y mujeres, mientras que un ensayo ${ }^{[14]}$ no reportó el sexo de sus participantes.

Todos los ensayos [10], [11], [12], [13], [14] compararon técnica CAD/CAM contra técnicas convencionales.

Dos ensayos $^{[12],[13]}$ usaron restauraciones tipo corona parcial unitaria inlays, onlays y overlays, y tres ${ }^{[10], ~[11], ~[14] ~}$ usaron restauraciones tipo corona total unitaria.

Dos ensayos ${ }^{[12], ~[13]}$ usaron sistema CEREC, uno ${ }^{[11]}$ evaluó el sistema Kavo Everest, uno ${ }^{[14]}$ usó sistema Yenadent $D$ 30 Eosint $M 270$, y uno ${ }^{[10]}$ no especificó el sistema CAD/CAM utilizado.

Para la técnica CAD/CAM, dos ensayos ${ }^{[10], ~[11]}$ usaron zirconia como material de casquete y recubrimiento, uno ${ }^{[14]}$ usó cromo - cobalto como material de casquete y cerámica como recubrimiento (no especifica material), uno ${ }^{[12]}$ usó feldespato reforzado con leucita como material de casquete y recubrimiento, y uno ${ }^{[13]}$ usó feldespato como material de casquete y recubrimiento.

Para la técnica convencional, tres ensayos ${ }^{[10],[11], ~[13] ~ u t i l i z a r o n ~ o r o ~ c o m o ~}$ material de casquete y recubrimiento Además, uno de ellos ${ }^{[13]}$ usó también Mirage y Empress. Un ensayo ${ }^{[12]}$ usó disilicato de litio como material de casquete y recubrimiento, y finalmente, un ensayo ${ }^{[14]}$ usó como material de casquete cromo - cobalto y cerámica como recubrimiento (no especifica material).

Los ensayos reportaron múltiples desenlaces, los cuales fueron agrupados por las revisiones sistemáticas de la siguiente manera:

- Fallo de restauración.

- Cambio de color superficial.

- Caries secundaria.

- Pérdida de vitalidad.

- Pérdida de retención.

- Fractura de casquete y/o recubrimiento.

- Extracción dentaria.

- USPHS score (Charlie / Delta).

- Discrepancia de adaptación marginal (medido en micrones).

Dos ensayos ${ }^{[11],[13]}$ tuvieron un seguimiento de cinco años, uno ${ }^{[10]}$ de cinco años y medio, uno ${ }^{[12]}$ de siete años, y uno ${ }^{[14]}$ no reportó tiempo de seguimiento.

* La información sobre los estudios primarios es extraída desde las revisiones sistemáticas identificadas, no directamente desde los estudios, a menos que se especifique lo contrario. 


\section{RESUMEN DE LOS RESULTADOS}

La información sobre los desempeños clínicos de restauraciones realizadas mediante técnica CAD/CAM en pacientes con indicación de restauración indirecta unitaria posterior está basada en cinco ensayos aleatorizados que incluyeron 513 dientes tratados.

Cuatro ensayos midieron el desenlace falla de restauración (473 dientes) [10], [11], [12], [13], tres ensayos midieron el desenlace pérdida de retención (393 dientes) [10], [11], [13], dos ensayos midieron el desenlace pérdida de vitalidad, extracción dentaria, caries radicular y cambio de color superficial (170 dientes) [10], [13]

El resumen de los resultados es el siguiente:

- Técnica CAD/CAM comparado con técnica convencional probablemente aumenta el riesgo de la falla de la restauración.

- Técnica CAD/CAM comparado con técnica convencional probablemente aumenta el riesgo de pérdida de retención.

- Técnica CAD/CAM comparado con técnica convencional podría disminuir levemente la pérdida de vitalidad (certeza de la evidencia baja).

- Técnica CAD/CAM comparado con técnica convencional podría resultar en poca o nula diferencia el riesgo de extracción dentaria (certeza de la evidencia baja).

- Técnica CAD/CAM comparado con técnica convencional podría resultar en poca o nula diferencia en el riesgo de desarrollar caries secundaria.

- Técnica CAD/CAM comparado con técnica convencional podría resultar en poca o nula diferencia en el cambio de color superficial.

\begin{tabular}{|c|c|c|c|c|}
\hline $\begin{array}{l}\text { Pacientes } \\
\text { Intervención } \\
\text { Comparación }\end{array}$ & $\begin{array}{l}\text { Pacientes con indicación } \\
\text { Restauración realizada } m \\
\text { Restauración realizada } m\end{array}$ & $\begin{array}{l}\text { ración indirect } \\
\text { écnica CAD/CA } \\
\text { écnica convenc }\end{array}$ & a posterior & \\
\hline \multirow{3}{*}{ Desenlaces } & \multicolumn{2}{|c|}{ Efecto absoluto* } & \multirow{3}{*}{$\begin{array}{l}\text { Efecto } \\
\text { relativo } \\
\text { (IC 95\%) }\end{array}$} & \multirow{3}{*}{$\begin{array}{l}\text { Certeza de la } \\
\text { evidencia } \\
\text { (GRADE) }\end{array}$} \\
\hline & $\begin{array}{c}\text { CON } \\
\text { técnica convencional } \\
\end{array}$ & $\begin{array}{c}\text { CON } \\
\text { CAD/CAM }\end{array}$ & & \\
\hline & Diferencia: $p$ & por 1000 & & \\
\hline \multirow{2}{*}{$\begin{array}{l}\text { Falla de la } \\
\text { restauración }\end{array}$} & 78 por 1000 & 152 por 1000 & \multirow{2}{*}{$\begin{array}{l}\text { RR } 1,96 \\
(1,11 \text { a } 3,47)\end{array}$} & \multirow{2}{*}{$\begin{array}{c}\oplus \oplus \oplus \bigcirc^{1} \\
\text { Moderado }\end{array}$} \\
\hline & $\begin{array}{l}\text { Diferencia: } \\
\text { (Margen de en }\end{array}$ & 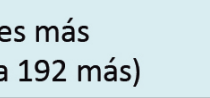 & & \\
\hline \multirow{2}{*}{$\begin{array}{l}\text { Pérdida de } \\
\text { retención }\end{array}$} & 44 por 1000 & 118 por 1000 & \multirow{2}{*}{$\begin{array}{l}\operatorname{RR} 2,68 \\
(1,35 \text { a } 5,33)\end{array}$} & \multirow{2}{*}{$\begin{array}{c}\oplus \oplus \oplus \bigcirc^{1} \\
\text { Moderado }\end{array}$} \\
\hline & \multicolumn{2}{|c|}{$\begin{array}{l}\text { Diferencia: } 74 \text { dientes más } \\
\text { (Margen de error: de } 15 \text { a } 190 \text { más) }\end{array}$} & & \\
\hline \multirow[b]{2}{*}{ Pérdida de vitalidad } & 29 por 1000 & 12 por 1000 & \multirow{2}{*}{$\begin{array}{l}\text { RR } 0,41 \\
(0,02 \text { a } 7,70)\end{array}$} & \multirow{2}{*}{$\stackrel{\oplus \oplus \bigcirc_{\mathrm{Baja}}^{1,2}}{ }$} \\
\hline & \multicolumn{2}{|c|}{$\begin{array}{l}\text { Diferencia: } 17 \text { dientes menos } \\
\text { (Margen de error: } 28 \text { menos a } 191 \text { más) }\end{array}$} & & \\
\hline \multirow[b]{2}{*}{ Extracción dentaria } & 10 por 1000 & 3 por 1000 & \multirow{2}{*}{$\begin{array}{l}\text { RR } 0,33 \\
(0,01 \text { a } 7,97)\end{array}$} & \multirow{2}{*}{$\underset{\text { Baja }}{\oplus \oplus \bigcirc^{1}}$} \\
\hline & \multicolumn{2}{|c|}{$\begin{array}{l}\text { Diferencia: } 7 \text { dientes menos } \\
\text { (Margen de error: } 9 \text { menos a } 66 \text { más) }\end{array}$} & & \\
\hline Caries secundaria & \multicolumn{3}{|c|}{$\begin{array}{l}\text { Dos ensayos [10],[13] reportaron que no hubo desarrollo de caries } \\
\text { secundaria ni en el grupo de intervención ni en el grupo de control. }\end{array}$} & $\begin{array}{c}\oplus \oplus \bigcirc \bigcirc^{1,2} \\
\text { Baja }\end{array}$ \\
\hline $\begin{array}{l}\text { Cambio de color } \\
\text { superficial }\end{array}$ & \multicolumn{3}{|c|}{$\begin{array}{l}\text { Dos ensayos [10],[13] reportaron que no hubo cambio de color } \\
\text { superficial ni en el grupo de intervención ni en el grupo de control. }\end{array}$} & $\underset{\mathrm{Baja}}{\oplus \oplus \bigcirc^{1,2}}$ \\
\hline \multirow{2}{*}{\multicolumn{5}{|c|}{$\begin{array}{l}\text { CAD/CAM: Computer-Aided Design/Diseño-Manufactura asistida por computadora } \\
\text { Margen de error: Intervalo de confianza del } 95 \% \text { (IC } 95 \%) \text {. } \\
\text { RR: Riesgo relativo. } \\
\text { DM: Diferencia de medias. } \\
\text { GRADE: Grados de evidencia del GRADE Working Group (ver más adelante). } \\
\text { *Los riesgos CON técnica convencional están basados en los riesgos del grupo control en los estudios. El riesgo CON } \\
\text { CAD/CAM (y su margen de error) está calculado a partir del efecto relativo (y su margen de error). }\end{array}$}} \\
\hline & & & & \\
\hline \multicolumn{5}{|c|}{$\begin{array}{l}{ }^{1} \text { Se disminuyó un nivel de certeza de evidencia por imprecisión, debido a que a cada extremo del intervalo de confianza las } \\
\text { decisiones clínicas variarán. En el caso del desenlace "extracción dentaria" se decidió disminuir dos niveles por imprecisión } \\
\text { debido a que cada extremo del intervalo de confianza conlleva a dos decisiones clínicas opuestas. Además, es esperable que } \\
\text { el resultado sea impreciso debido al tamaño muestral y a la poca cantidad de eventos observados en los ensayos. }\end{array}$} \\
\hline \multicolumn{5}{|c|}{$\begin{array}{l}{ }^{2} \text { Se disminuyó un nivel de certeza de evidencia por riesgo de sesgo, debido a que no estaba claro el ocultamiento de la } \\
\text { secuencia de aleatorización. }\end{array}$} \\
\hline
\end{tabular}


Siga el enlace para acceder a la versión interactiva de esta tabla (Interactive Summary of Findings - iSoF)

\begin{tabular}{l}
\hline Acerca de la certeza de la evidencia (GRADE)* \\
\hline$\oplus \oplus \oplus \oplus$ \\
Alta: La investigación entrega una muy buena indicación del efecto probable. La probabilidad de \\
que el efecto sea sustancialmente distinto† es baja. \\
$\oplus \oplus \oplus \bigcirc$ \\
Moderada: La investigación entrega una buena indicación del efecto probable. La probabilidad de \\
que el efecto sea sustancialmente distinto† es moderada. \\
$\oplus \oplus \bigcirc$ \\
Baja: La investigación entrega alguna indicación del efecto probable. Sin embargo, la probabilidad \\
de que el efecto sea sustancialmente distinto† es alta. \\
$\oplus \bigcirc \bigcirc \bigcirc$ \\
Muy baja: La investigación no entrega una estimación confiable del efecto probable. La \\
probabilidad de que el efecto sea sustancialmente distinto† es muy alta. \\
\hline *Esto es también denominado 'calidad de la evidencia' o 'confianza en los estimadores del efecto'. \\
†Sustancialmente distinto = una diferencia suficientemente grande como para afectar la decisión \\
\hline
\end{tabular}

\section{OTRAS CONSIDERACIONES PARA LA TOMA DE DECISIÓN}

\section{A quién se aplica y a quién no se aplica esta evidencia}

- Los resultados de este resumen son aplicables a todos los pacientes que requieran una restauración indirecta unitaria posterior en dientes permanentes.

- Si bien los ensayos incluyen a pacientes con promedios de edad mayores a 18 años, no existen razones clínicas para no extrapolar los resultados a pacientes menores en dentición permanente.

- Los resultados de este resumen no aplican para pacientes que requieran una restauración indirecta unitaria anterior, múltiple anterior o posterior, implantosoportadas o sobre dientes temporales.

\section{Sobre los desenlaces incluidos en este resumen}

- Los desenlaces seleccionados son considerados críticos para la toma de decisión de acuerdo a la opinión de los autores de este resumen, coincidiendo en general con los evaluados por las revisiones sistemáticas identificadas.

- El desenlace falla de la restauración es crítico para la toma de decisión en salud, ya que muestra el comportamiento clínico de la restauración realizada por una técnica u otra.

- Los desenlaces "número de sesiones" y "sensibilidad postoperatoria" no fueron reportados en los ensayos, pero son considerados factores importantes para la toma de decisión de acuerdo a la opinión de los autores de este resumen.

\section{Balance daño/beneficio y certeza de la evidencia}

- La evidencia muestra un beneficio en el uso de la técnica convencional en comparación con la técnica CAD/CAM, dado que esta última probablemente aumenta la falla de restauración e incrementa la pérdida de retención.

- Por otra parte, la técnica CAD/CAM en comparación con la técnica convencional podría disminuir levemente la pérdida de vitalidad y extracción dentaria, además de producir poca o nula diferencia entre las técnicas en la presencia de caries secundaria y cambio de color superficial, pero la certeza de la evidencia ha sido evaluada como baja.

- Considerando lo anterior, el balance daño/beneficio podría estar a favor de realizar la técnica convencional por sobre la técnica CAD/CAM.

\section{Consideraciones de recursos}

- Ninguna de las revisiones identificadas realizó un análisis de costos entre las técnicas convencionales y CAD/CAM.

- Existe una diferencia considerable de costos en restauraciones indirectas realizadas con técnica CAD/CAM y convencional, siendo la CAD/CAM más costosa. No obstante, el tiempo requerido para el sistema CAD/CAM es considerablemente menor al de sistemas convencionales ${ }^{[2]}$, pues no requiere de una etapa de laboratorio para su confección, lo que disminuye el número de sesiones que se necesitan para la rehabilitación.

- Se requieren estudios que evalúen el costo final de una restauración indirecta realizada con sistemas CAD/CAM y convencionales, considerando el precio y el número de sesiones requeridas.

\section{Qué piensan los pacientes y sus tratantes}

- Enfrentados a la evidencia presentada en este resumen, la mayoría de los tratantes y pacientes deberían inclinarse a favor de las técnicas convencionales para realizar restauraciones indirectas unitarias posteriores, debido a su mejor desempeño clínico comparado con la técnica CAD/CAM.

- Sin embargo, se ha visto que los pacientes muestran preferencia por la técnica CAD/CAM debido al menor tiempo de confección ${ }^{[2]}$, a que el software permite visualizar un diseño digital previo al tallado de la restauración, involucrándolos en la toma de decisión, y a que la impresión digital es menos invasiva y más cómoda para el paciente ${ }^{[3]}$.

- En la práctica clínica, también se observa variabilidad en las preferencias de los tratantes. Por un lado, hay quienes prefieren la técnica CAD/CAM dado que al tratarse de un proceso automatizado, minimizaría la probabilidad de error humano[6], ofrece una mayor versatilidad en el diseño de la restauración en un menor tiempo de confección ${ }^{[2]}$ y no requiere de etapas analógicas como la toma de impresión y provisionalización, ni de coordinación con un laboratorio ${ }^{[3]}$. Por otro lado, el uso de la técnica convencional no supone una gran inversión inicial en equipo especializado ni una curva de aprendizaje para su uso ${ }^{[4]}$.

- En consideración de lo anterior, es importante que la toma de decisión se produzca en conjunto con los pacientes, informándoles sobre las limitaciones y conclusiones de la evidencia existente.

\section{Diferencias entre este resumen y otras fuentes}

- Las conclusiones de este resumen concuerdan con dos revisiones

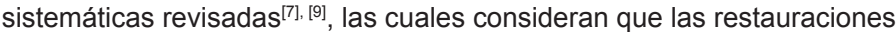
confeccionadas con la técnica CAD/CAM tienen menor longevidad, influenciado por el tipo de material y los diferentes procesos utilizados.

- Una revisión sistemática ${ }^{[8]}$ no concuerda con las conclusiones de este resumen, al considerar que las restauraciones confeccionadas con la técnica CAD/CAM tienen igual tasa de supervivencia comparadas con la técnica convencional.

- No se identificaron guías clínicas que recomiendan el uso de una técnica sobre otra en la población de interés.

\section{¿Puede que cambie esta información en el futuro?}

- En general, es improbable que futuras investigaciones cambien las conclusiones de este resumen. Sólo los desenlaces pérdida de vitalidad y extracción dentaria presentan una baja certeza de la evidencia, por lo que es probable que éstas cambien.

- Identificamos un ensayo clínico aleatorizado en curso ${ }^{[18]}$ en U.S national library of medicine evaluando la efectividad de las restauraciones con técnica CAD/CAM comparada con la técnica convencional.

- Identificamos dos revisiones sistemáticas en curso ${ }^{[19],[20]}$ en International prospective register of systematic reviews (PROSPERO) evaluando la pregunta de interés. 


\section{CÓMO REALIZAMOS ESTE RESUMEN}

Mediante métodos automatizados y colaborativos recopilamos toda la evidencia relevante para la pregunta de interés y la presentamos en una matriz de evidencia.

Siga el enlace para acceder a la versión interactiva: Técnica CAD/CAM comparado con técnica convencional en pacientes con indicación de restauración indirecta unitaria posterior

\section{NOTAS}

Si con posterioridad a la publicación de este resumen se publican nuevas revisiones sistemáticas sobre este tema, en la parte superior de la matriz se mostrará un aviso de "nueva evidencia".

Este artículo es parte del proyecto síntesis de evidencia de Epistemonikos. Se elabora con una metodología preestablecida, siguiendo rigurosos estándares metodológicos y proceso de revisión por pares interno. Cada uno de estos artículos corresponde a un resumen, denominado FRISBEE (Friendly Summary of Body of Evidence using Epistemonikos), cuyo principal objetivo es sintetizar el conjunto de evidencia de una pregunta específica, en un formato amigable a los profesionales clínicos. Sus principales recursos se basan en la matriz de evidencia de Epistemonikos y análisis de resultados usando metodología GRADE. Mayores detalles de los métodos para elaborar este FRISBEE están descritos aquí (http://dx.doi.org/10.5867/medwave.2014.06.5997)

La Fundación Epistemonikos es una organización que busca acercar la información a quienes toman decisiones en salud, mediante el uso de tecnologías. Su principal desarrollo es la base de datos Epistemonikos (www.epistemonikos.org).

\section{DECLARACIÓN DE CONFLICTOS DE INTERESES}

Los autores declaran no tener conflictos de intereses con la materia de este artículo.

\section{AGRADECIMIENTOS}

Este resumen de evidencia fue elaborado con el apoyo metodológico del Centro Evidencia UC, Facultad de Medicina, Pontificia Universidad Católica de Chile.

\section{Bibliografía}

1. da Veiga AMA, Cunha AC, Ferreira DMTP. Longevity of direct and indirect resin composite restorations in permanent posterior teeth: A systematic review and metaanalysis. J Dent. 2016;54:1-12.

2. Witkowski S. Computer-aided design and fabrication of dental restoration. Current systems and future possibilities. JADA. 2006;137(9):1289-96.

3. Mehl A, Gloger W, Hickel R. Posibilidades y perspectivas del nuevo sistema de exploración PRECISAN $囚$, para la tecnología CAD/CAM. Quintessence técnica (ed. esp) 1999;10(8):454-459.

4. Trost L, Stines S, Burt L. Making informed decisions about incorporating a CAD/ CAM system into dental practice. JADA 2006;137(9 supplement):32S-36S.

5. Petya K. Brawek SW, Lutz Endres, Armin Kirsten, Sven Reich. The clinical accuracy of single crowns exclusively fabricated by digital workflow-the comparison of two systems. Clin Oral Invest. 2013;17:2119-25.

6. Gómez Cogolludo P, Suárez, MJ, Peláez, J, López Lozano, JF. Caso clínico:

prótesis parcial fija posterior con el sistema Lava.. Rev Int Prot Estomatol.

2008;10(1):11-6

7. Rodrigues SB, Franken P, Celeste RK, Leitune VCB, Collares FM. CAD/CAM or conventional ceramic materials restorations longevity: a systematic review and meta-analysis. J Prosthodont Res. 2019 Oct;63(4):389-395. doi: 10.1016/j. jpor.2018.11.006. Epub 2019 Jul 11. PMID: 31303569.

8. Alves de Carvalho IF, Santos Marques TM, Araújo FM, Azevedo LF, Donato $\mathrm{H}$, Correia A. Clinical Performance of CAD/CAM Tooth-Supported Ceramic Restorations: A Systematic Review. Int J Periodontics Restorative Dent. 2018 Jul/ Aug;38(4):e68-e78. doi: 10.11607/prd.3519. PMID: 29889916.

9. Papadiochou S, Pissiotis AL. Marginal adaptation and CAD-CAM technology: A systematic review of restorative material and fabrication techniques. J Prosthet Dent. 2018 Apr;119(4):545-551. doi: 10.1016/j.prosdent.2017.07.001. Epub 2017 Sep 28. PMID: 28967399.

10. Monaco C, Llukacej A, Baldissara P, Arena A, Scotti R. Zirconia-based versus metal-based single crowns veneered with overpressing ceramic for restoration of posterior endodontically treated teeth: 5-year results of a randomized controlled clinical study. J Dent. 2017 Oct;65:56-63. doi: 10.1016/j.jdent.2017.07.004. Epub 2017 Jul 21. PMID: 28736293.

11. Passia N, Stampf S, Strub JR. Five-year results of a prospective randomised controlled clinical trial of posterior computer-aided design-computer-aided manufacturing ZrSiO4 -ceramic crowns. J Oral Rehabil. 2013 Aug;40(8):609-17. doi: 10.1111/joor.12075. Epub 2013 Jun 8. PMID: 23745725.

12. Guess PC, Selz CF, Steinhart YN, Stampf S, Strub JR. Prospective clinical splitmouth study of pressed and CAD/CAM all-ceramic partial-coverage restorations: 7-year results. Int J Prosthodont. 2013 Jan-Feb;26(1):21-5. doi: 10.11607/ijp.3043. PMID: 23342329.

13. Molin MK, Karlsson SL. A randomized 5-year clinical evaluation of 3 ceramic inlay systems. Int J Prosthodont. 2000 May-Jun;13(3):194-200. PMID: 11203631. 14. Tamac E, Toksavul S, Toman M. Clinical marginal and internal adaptation of CAD/CAM milling, laser sintering, and cast metal ceramic crowns. J Prosthet Dent. 2014 Oct;112(4):909-13. doi: 10.1016/j.prosdent.2013.12.020. Epub 2014 May 10. PMID: 24819532.

15. Federlin M, Hiller KA, Schmalz G. Controlled, prospective clinical split-mouth study of cast gold vs. ceramic partial crowns: 5.5 year results. Am J Dent. 2010 Jun;23(3):161-7. PMID: 20718214.

16. Vigolo P, Mutinelli S. Evaluation of zirconium-oxide-based ceramic single-unit posterior fixed dental prostheses (FDPs) generated with two CAD/CAM systems compared to porcelain-fused-to-metal single-unit posterior FDPs: a 5-year clinical prospective study. J Prosthodont. 2012 Jun;21(4):265-9. doi: 10.1111/j.1532849X.2011.00825.x. Epub 2012 Feb 19. PMID: 22339945.

17. Huang Z, Zhang L, Zhu J, Zhang X. Clinical marginal and internal fit of metal ceramic crowns fabricated with a selective laser melting technology. J Prosthet Dent. 2015 Jun;113(6):623-7. doi: 10.1016/j.prosdent.2014.10.012. Epub 2015 Mar 18 PMID: 25794918.

18. Azerad J., Comparison of Conventional and CAD/CAM Dental Restaurations (EVACFAOD). ClinicalTrials.gov 2017 Oct.

19. Oen K., Veitz - Keenan A. CAD/CAM versus traditional indirect methods in the fabrication of inlays, onlays, and crowns [Cochrane Protocol]. International prospective register of systematic reviews. 2015 Mar.

20. Afrashtehfar K., Treviño-Santos A. A systematic review of clinical outcomes of pressable versus machinable lithium disilicate restorations. International prospective register of systematic reviews. $2016 \mathrm{Apr}$. 